\title{
Reductive Removal of the Pivaloyl Protecting Group from Tetrazoles by a Naphthalene-Catalysed Lithiation Process
}

\author{
Cherif Behloul, ${ }^{\mathrm{a}}{ }^{\mathrm{a}}$ Aicha Chouti, ${ }^{\mathrm{a}}$ David Guijarro, ${ }^{\mathrm{b}}$ Carmen Nájera ${ }^{\mathrm{b}}$ and Miguel Yus ${ }^{\mathrm{b}}$ \\ ${ }^{a}$ Laboratoire des Produits Naturels d’origine Végétale et de Synthèse Organique, Université de Constantine -I- 25000- Algérie \\ ${ }^{\text {b}}$ Departamento de Química Orgánica, Facultad de Ciencias and Instituto de Síntesis Orgánica (ISO), Universidad de Alicante, Apdo. 99, \\ 03080 Alicante (Spain)
}

Tel/Fax: 21331818862

Email: afiza72@gmail.com

Received: The date will be inserted once the manuscript is accepted.

\begin{abstract}
The reaction of different tetrazoles protected with a pivaloyl group, with an excess of lithium and a catalytic amount of naphthalene (8 mol\%) led, after treatment with methanol, to the corresponding free tetrazoles through a reductive carbon-nitrogen bond cleavage. This methodology represents a reasonable alternative to other non-reductive protocols.
\end{abstract}

Keywords: tetrazoles, pivaloyl, lithium.

The pivaloyl group has been widely used in organic synthesis to protect alcohols, amines and thiols, due to its easy introduction, its stability under a variety of reaction conditions and its relatively easy removal to give the corresponding depivalated compounds. ${ }^{1}$ Among the different methodologies reported to achieve the deacylation of protected alcohols and amines, we can find hydrolysis under basic or acidic conditions, ${ }^{2}$ reduction with alkali metals in liquid ammonia, ${ }^{3}$ reductive cleavage using an arene-catalysed lithiation, ${ }^{4}$ reaction with hydride sources, ${ }^{5}$ electrolysis, ${ }^{6}$ and enzymatic methods. ${ }^{7}$

On the other hand, the tetrazole moiety is present in several biologically active compounds, such as sartans, which are pharmaceuticals that have proved to be efficient for the treatment of hypertension, kidney damage caused by diabetes and heart failure. Usually, the synthesis of sartans involves steps of protection and deprotection of the tetrazole ring.8 The effective protection caused by the high steric demand of the pivaloyl group, together with the ease of its introduction into and its removal from the nitrogen atom, are features that could make the pivaloyl group to become a very useful tool for the preparation of such an interesting family of drugs. Thus, the development of procedures that could remove the pivaloyl protecting group without affecting the tetrazole ring would be very interesting. ${ }^{8}$

In the last years our research group has been using an arene-catalysed lithiation to perform metallations under very mild reaction conditions. ${ }^{9-12}$ This lithiation methodology has been applied to the reductive cleavage of trityl ethers $^{13}$ and amines, ${ }^{14}$ for the desilylation of silylated alcohols, amines and thiols, ${ }^{15}$ for the cleavage of carbonates, carbamates and thiocarbonates, ${ }^{16}$ and for the deacylation of esters, thioesters and amides. ${ }^{17}$

In this paper we wish to report the use of a naphthalenecatalysed lithiation to perform the removal of the pivaloyl protecting group from tetrazoles under very mild conditions.
The reaction of different N-pivaloyl tetrazoles 1a-1f with an excess of lithium powder (1:9 molar ratio) and a catalytic amount of naphthalene (1:0.16 molar ratio) in THF at $0{ }^{\circ} \mathrm{C}$ for $3 \mathrm{~h}$ led, after quenching with methanol, to the corresponding free tetrazoles $\mathbf{2 a - 2 f}$ (Table 1, entries 16).

$\mathrm{i}, \mathrm{Li}, \mathrm{C}_{10} \mathrm{H}_{8}(8 \mathrm{~mol} \%)$
$\mathrm{ii}, \mathrm{MeOH}, 0$ to $20^{\circ} \mathrm{C}$
$\mathrm{R}=$ alkyl, aryl, benzyl
$\mathrm{Pv}=t-\mathrm{BuCO}$

\section{Equation 1}

The deprotected tetrazoles were isolated generally in good yields (Equation 1 and Table 1). In general, moderate to good yields were obtained in the deprotection of $\mathrm{N}$-pivaloyl tetrazoles bearing aromatic (Table 1, entries 1 and 2), aliphatic (Table 1, entries 3 and 5) or benzylic (Table 1, entry 6) R groups. Interestingly, a substituent functionalyzed with a carbonyl group, which could undergo reduction under these reaction conditions, was not altered and the depivalated product was obtained in $62 \%$ yield (Table 1, entry 4). All the lithiation processes were complete in a reaction time of $3 \mathrm{~h}$.

The starting tetrazoles were prepared by reaction of sodium azide with the corresponding nitriles in the presence of an amine. The isolated tetrazoles were then pivalated on the $\mathrm{N} 1$ by reaction with pivaloyl chloride, giving the expected protected tetrazoles 1 in good yields

In conclusion, we have reported in this paper an efficient method to remove the pivaloyl protecting group from different tetrazoles bearing aliphatic, aromatic or benzylic substituents on the carbon atom of the ring, under very mild reaction conditions. This procedure is a reasonable alternative to other methodologies, especially those involving hydrolysis, which usually require harsh reaction conditions.

FT-IR spectra were obtained on a Nicolet Impact 400D spectrophotometer using $\mathrm{KBr}$ plates (for solid compounds). NMR spectra were recorded on a Bruker spectrometer (400 MHz for ${ }^{1} \mathrm{H}$ and $100 \mathrm{MHz}$ for ${ }^{13} \mathrm{C}$ ) using $\mathrm{CDCl}_{3}, \mathrm{DMSO}-\mathrm{d}_{6}, \mathrm{MeOD}-\mathrm{d}_{4}$ as solvents and TMS (0.00 
ppm, $\left.{ }^{1} \mathrm{H}\right)$ and $\mathrm{CDCl}_{3}\left(77.0 \mathrm{ppm},{ }^{13} \mathrm{C}\right)$, DMSO-d $\mathrm{d}_{6}(2.50$ ppm, ${ }^{1} \mathrm{H}$ and $\left.39.75 \mathrm{ppm},{ }^{13} \mathrm{C}\right)$, MeOD- $\mathrm{d}_{4}\left(4.87 \mathrm{ppm},{ }^{1} \mathrm{H}\right.$ and $49.0 \mathrm{ppm},{ }^{13} \mathrm{C}$ ) as internal standards; chemical shifts are given in $\delta(\mathrm{ppm})$ and coupling constants $(J)$ in $\mathrm{Hz}$. Elemental analysis were measured by the Technical Services at the University of Alicante. Column chromatography was performed using silica gel 60 (35-70 mesh) or basic aluminium oxide (50-160 micrometres particle size). Lithium powder was prepared according to the procedure described in reference. ${ }^{18}$ Commercially available $n$-butyllithium was titrated with a $1 \mathrm{M}$ solution of sec-butanol in xylene using 1,10-phenanthroline as indicator. ${ }^{19}$ Commercially available anhydrous THF (99.9\%, water content $\leq 0.006 \%$, Acros) was used as solvent in all the lithiation reactions.

Table 1 Reductive Removal of the Pivaloyl Group from Protected Tetrazoles 1

Entry Substrate<smiles>CC(C)(C)C(=O)n1nnnc1-c1ccccc1</smiles>

$1 \mathrm{a}$

2<smiles>Cc1ccc(-c2ccccc2-c2nnnn2C(=O)C(C)(C)C)cc1</smiles>

1b

3<smiles>CC(C)(C)C(=O)n1nnnc1C(C)(C)C</smiles>

1c

4<smiles>CC(C)(C)C(=O)Cc1nnnn1C(=O)C(C)(C)C</smiles>

$1 d$

5<smiles>Cc1nnnn1C(=O)C(C)(C)C</smiles>

6<smiles>CC(C)(C)C(=O)n1nnnc1C(c1ccccc1)c1ccccc1</smiles>

1f
Time

3h

Product<smiles>c1ccc(-c2nnn[nH]2)cc1</smiles>

Yield (\%)

2a<smiles>Cc1ccc(-c2ccccc2-c2nnn[nH]2)cc1</smiles>

56

2b<smiles>CC(C)(C)c1nnn[nH]1</smiles>

2c<smiles>[Z10]C(C)(C)C(=O)Cc1nnn[nH]1</smiles>

62

61<smiles></smiles>

$3 \mathrm{~h}$<smiles>c1ccc(C(c2ccccc2)c2nnn[nH]2)cc1</smiles>

61

2f 
${ }^{\text {a }}$ Yield of isolated product after extraction and recrystallization, based on the starting material $\mathbf{1}$

All reagents used for the synthesis of substrates $\mathbf{1}$ and naphthalene were commercially available (Acros, Aldrich) and were used without further purification. All glassware was dried in an oven at $100^{\circ} \mathrm{C}$ and cooled to room temperature under argon before use.

\section{Synthesis of tetrazoles. General Procedure ${ }^{20}$}

The mixture of a nitrile (50 mmol), $\mathrm{NaN}_{3}(65 \mathrm{mmol})$ and an amine salt $\mathrm{Et}_{3} \mathrm{~N} \cdot \mathrm{HCl}(150 \mathrm{mmol})$ in toluene (100 $\mathrm{mL}$ ) was heated to $110^{\circ} \mathrm{C}$ with stirring for $24 \mathrm{~h}$ for nitriles with aromatic R groups (2a and $2 \mathrm{f}$ ) and for 17 and $30 \mathrm{~h}$, respectively, in the case of nitriles bearing aliphatic groups (2c and $2 \mathrm{~d}$ ). After cooling to room temperature, the mixture was extracted with water $(100 \mathrm{~mL}) .36 \%$ $\mathrm{HCl}$ was added dropwise to the aqueous layer till $\mathrm{pH}$ was acidic. After filtration, the solid was dried under reduced pressure, yielding the expected tetrazoles 2 . The corresponding physical and spectroscopic data for all tetrazoles 2 follow.

\section{5-phenyl-1H-tetrazole (2a)}

White solid; yield: 95\%; mp: $215-216^{\circ} \mathrm{C}$.

${ }^{1} \mathrm{H}$ NMR (400 MHz, DMSO-d $\left.\mathrm{d}_{6}\right): \delta=7.55-7.62$ (m, 3H), 8.01-8.07 (m, 2H).

${ }^{13} \mathrm{C}$ NMR $\left(100 \mathrm{MHz}, \mathrm{DMSO}-\mathrm{d}_{6}\right): \delta=124.2(2 \times \mathrm{CH})$, 127.0 (C), 129.4 (CH), 131.3 (2×CH), $155.3(\mathrm{C})$.

5-(4'-methyl-[1,1'-biphenyl]-2-yl)-1H-tetrazole (2b) ${ }^{21}$ Brown solid; yield: $78 \%$; mp: $149-151^{\circ} \mathrm{C}$.

IR (KBr): 3336, 2974, 2900, 1080, 1046, 879, $755 \mathrm{~cm}^{-1}$.

${ }^{1} \mathrm{H}$ NMR (400 MHz, DMSO-d ${ }_{6}$ ): $\delta=2.28$ (s, 3H), 6.98 (d, $J=8.0 \mathrm{~Hz}, 2 \mathrm{H}), 7.12$ (d, $J=8.0 \mathrm{~Hz}, 2 \mathrm{H}), 7.55$ (ddd, $J=10.3,5.8,1.9 \mathrm{~Hz}, 2 \mathrm{H}), 7.63-7.69$ (m, 2H).

${ }^{13} \mathrm{C}$ NMR (100 MHz, DMSO-d $\left.{ }_{6}\right): \delta=20.7\left(\mathrm{CH}_{3}\right), 123.4$ (CH), $127.5(\mathrm{CH}), 128.7(2 \times \mathrm{CH}), 128.9(\mathrm{CH}), 130.5$ (2×CH), $130.6(\mathrm{CH}), 131.1(\mathrm{C}), 136.3(\mathrm{C}), 136.8(\mathrm{C})$, 141.5 (C), 155.1 (C).

Anal. Calcd for $\mathrm{C}_{14} \mathrm{H}_{12} \mathrm{~N}_{4}$ : C, 71.17; H, 5.12; N, 23.71 . Found: C, 71.19; H, 5.10; N, 23.74.

\section{5-(tert-butyl)-1H-tetrazole (2c) ${ }^{21}$}

White solid; yield: 90\%; mp: $208-210^{\circ} \mathrm{C}$.

IR (KBr): 2986, 2977, 2869, 1717, 1558, 1366, 1066, $1045,1008 \mathrm{~cm}^{-1}$.

${ }^{1} \mathrm{H}$ NMR (400 MHz, DMSO-d $\mathrm{d}_{6}$ ): $\delta=1.35$ (s, 9H).

${ }^{13} \mathrm{C}$ NMR (100 MHz, DMSO-d $\left.\mathrm{d}_{6}\right): \delta=28.9\left(3 \times \mathrm{CH}_{3}\right), 30.3$ (C), 163.4 (C).

\section{3,3-dimethyl-1-(1H-tetrazol-5-yl)butan-2-one (2d)}

Orange solid; yield: $85 \%$; mp: $152-154^{\circ} \mathrm{C}$.

IR (KBr): 2973, 2322, 1716, 1365, 1048, 1007, $728 \mathrm{~cm}^{-}$ 1 .
${ }^{1} \mathrm{H}$ NMR (400 MHz, DMSO-d 6 ): $\delta=1.18$ (s, 9H), 4.41 (s, 2H).

${ }^{13} \mathrm{C}$ NMR (100 MHz, DMSO-d $\left.\mathrm{d}_{6}\right): \delta=25.8\left(3 \times \mathrm{CH}_{3}\right), 32.2$ $\left(\mathrm{CH}_{2}\right), 44.0$ (C-C=O), $128.2(\mathrm{C}), 209.3(\mathrm{C}=\mathrm{O})$.

Anal. Calcd for $\mathrm{C}_{7} \mathrm{H}_{12} \mathrm{~N}_{4} \mathrm{O}$ : C, 49.99; H, 7.19; N, 33.31. Found: C, 49.97; H, 7.16; N, 33.28.

\section{5-methyl-1H-tetrazole (2e)}

White solid; yield: 93\%; mp: $138-140^{\circ} \mathrm{C}$.

IR (KBr): 3005, 2879, 2605, 1578, 1565, 1112, 1052, 899, $683 \mathrm{~cm}^{-1}$.

${ }^{1} \mathrm{H}$ NMR (400 MHz, DMSO-d $\left.\mathrm{d}_{6}\right): \delta=2.46$ (s, 3H).

${ }^{13} \mathrm{C}$ NMR (100 MHz, DMSO-d $\left.\mathrm{d}_{6}\right): \delta=8.4\left(\mathrm{CH}_{3}\right), 152.2$ (C).

Anal. Calcd for $\mathrm{C}_{2} \mathrm{H}_{4} \mathrm{~N}_{4}$ : C, 28.57; H, 4.80; N, 66.64. Found: C, 28.60; H, 4.78; N, 66.165.

\section{5-benzhydryl-1H-tetrazole (2f)}

White solid; yield: $72 \%$; mp: $165-166^{\circ} \mathrm{C}$.

IR (KBr): 3264, 2917, 1560, 1446, 743, 695, $632 \mathrm{~cm}^{-1}$.

${ }^{1} \mathrm{H}$ NMR (400 MHz, MeOD-d ${ }_{4}$ ): $\delta=5.85$ (s, 1H), 7.14$7.30(\mathrm{~m}, 10 \mathrm{H})$.

${ }^{13} \mathrm{C}$ NMR (100 MHz, MeOD-d 4$): \delta=40.8(\mathrm{CH}), 128.6$ ( $2 \times \mathrm{CH}), 129.6(4 \times \mathrm{CH}), 129.9(4 \times \mathrm{CH}), 140.8(\mathrm{C}), 160.0$ $(2 \times \mathrm{C})$.

Anal. Calcd for $\mathrm{C}_{14} \mathrm{H}_{12} \mathrm{~N}_{4}$ : C, 71.17; H, 5.12; N, 23.71. Found: C, 71.14; H, 5.16; N, 23.74.

\section{Synthesis of the Protected Tetrazoles 1. General Pro- cedure}

$n$-BuLi (4 mL of a 2.5 M solution in hexane, $10.0 \mathrm{mmol}$ ) was dropwise added to a stirred solution of the corresponding tetrazole $(10.0 \mathrm{mmol})$ in anhydrous THF (10 $\mathrm{mL}$ ) under Ar at $0{ }^{\circ} \mathrm{C}$. 10 Minutes after the addition had been completed, pivaloyl chloride (1.23 mL, $10.0 \mathrm{mmol}$ ) was added during ca. $5 \mathrm{~min}$. After stirring at room temperature overnight, the reaction was quenched with $\mathrm{H}_{2} \mathrm{O}$ $(5 \mathrm{~mL})$ and extracted with EtOAc $(3 \times 15 \mathrm{~mL})$ and the combined organic phases were washed with brine $(5 \mathrm{~mL})$ and dried $\left(\mathrm{Na}_{2} \mathrm{SO}_{4}\right)$. After filtration and evaporation of the solvents, the resulting residue was purified by recrystallisation (hexane-EtOAc) affording the expected $\mathrm{N}$ pivaloyltetrazoles $\mathbf{1}$ in pure form. Their corresponding physical, spectroscopic and analytical data follow.

\section{2,2-dimethyl-1-(5-phenyl-1H-tetrazol-1-yl)propan- 1-one (1a)}

White solid; yield: $65 \%$ (1.49 g); mp: $218-220^{\circ} \mathrm{C}$.

IR (KBr): 1701, 1608, 1562, 1484, 1409, 1256, 685. 
${ }^{1} \mathrm{H}$ NMR (400 MHz, MeOD-d 4 ): $\delta=1.06(\mathrm{~s}, 9 \mathrm{H})$, 7.45-7.90 (m, 5H).

${ }^{13} \mathrm{C}$ NMR $\left(100 \mathrm{MHz}, \mathrm{MeOD}-\mathrm{d}_{4}\right): \delta=27.6\left(3 \times \mathrm{CH}_{3}\right)$, 39.3 (C), 125.6 (C), $128.2(2 \times \mathrm{CH}), 130.5(2 \times \mathrm{CH})$, 132.5 (CH), 157.7 (C).

Anal for $\mathrm{C}_{12} \mathrm{H}_{14} \mathrm{~N}_{4} \mathrm{O}$ : C, 62.69; $\mathrm{H}, 6.13 ; \mathrm{N}, 24.33$. Found: C, 62.66; H, 6.15; N, 24.37.

\section{2,2-dimethyl-1-[5-(4'-methyl-1,1'-biphenyl-2-yl)-1 $H$ -} tetrazol-1-yl]propan-1-one (1b)

Yellow solid; yield: 62\% (0.072 g).

IR (KBr): 1712, 1482, 1244, 1078, 823, $754 \mathrm{~cm}^{-1}$.

${ }^{1} \mathrm{H}$ NMR (400 MHz, MeOD-d $)_{4}$ : $\delta=0.92(\mathrm{~s}, 9 \mathrm{H})$, 2.27 (s, 3H), 6.93-7.08 (m, 4H), 7.45-7.63 (m, 4H).

${ }^{13} \mathrm{C}$ NMR $\left(100 \mathrm{MHz}, \mathrm{MeOD}-\mathrm{d}_{4}\right): \delta=21.1\left(\mathrm{CH}_{3}\right), 27.6$ $\left(3 \times \mathrm{CH}_{3}\right), 124.2(\mathrm{C}), 128.7,129.9,130.2,131.6,131.8$, $132.5(8 \times \mathrm{CH}), 137.6(\mathrm{C}), 138.8(2 \times \mathrm{C}), 143.6(2 \times \mathrm{C})$ 156.8 (C).

Anal for $\mathrm{C}_{19} \mathrm{H}_{20} \mathrm{~N}_{4} \mathrm{O}$ : C, 71.23; H, 6.29; N, 17.49. Found: C, 71.23; H, 6.32; N, 17.53.

\section{1-[5-(tert-butyl)-1H-tetrazol-1-yl]-2,2- dimethylpropan-1-one (1c)}

White solid; yield: $70 \%$ (0.032 g); mp: $104-106^{\circ} \mathrm{C}$.

IR (KBr): 1732, 1264, 1218, 1045, 703.

${ }^{1} \mathrm{H}$ NMR (400 MHz, MeOD-d $\mathrm{d}_{4}$ ): $\delta=1.42$ (s, 18H).

${ }^{13} \mathrm{C}$ NMR $\left(100 \mathrm{MHz}, \mathrm{MeOD}-\mathrm{d}_{4}\right): \delta=29.5\left(3 \times \mathrm{CH}_{3}\right)$, $31.8\left(3 \times \mathrm{CH}_{3}\right), 165.7$ (C), 214.1 (CO).

Anal for $\mathrm{C}_{10} \mathrm{H}_{18} \mathrm{~N}_{4} \mathrm{O}$ : C, 57.12; H, 8.63; N, 26.64. Found: C, 57.12; H, 8.62; N, 26.66.

\section{3,3-dimethyl-1-(1-pivaloyl-1H-tetrazol-5-yl)butan-2- one (1d)}

Brown solid; yield: 80\% (1.18 g).

${ }^{1} \mathrm{H}$ NMR (400 MHz, MeOD- $\mathrm{d}_{4}$ ): $\delta=2.74$ (s, 18H), $2.80(\mathrm{~s}, 2 \mathrm{H})$.

${ }^{13} \mathrm{C}$ NMR (100 MHz, MeOD-d $\left.\mathrm{d}_{4}\right): \delta=26.4,26.8,27.5$ $\left(3 \times \mathrm{CH}_{3}\right), 27.6,27.7,27.9\left(3 \times \mathrm{CH}_{3}\right), 31.7\left(\mathrm{CH}_{2}\right), 39.3$ (C), $45.5(\mathrm{C}), 152.5(\mathrm{C}), 182.5(\mathrm{C}=\mathrm{O}), 210.1(\mathrm{C}=\mathrm{O})$.

Anal for $\mathrm{C}_{12} \mathrm{H}_{20} \mathrm{~N}_{4} \mathrm{O}_{2}$ : C, 57.12; H, 7.99; N, 22.21. Found: C, 57.10; H, 8.01; N, 22.24.

\section{2,2-dimethyl-1-(5-methyl-1H-tetrazol-1-yl)propan-1- one (1e)}

White solid; yield: $85 \%$ (1.43 g); mp: $152-157^{\circ} \mathrm{C}$.

${ }^{1} \mathrm{H}$ NMR (400 MHz, MeOD- $\mathrm{d}_{4}$ ): $\delta=0.92$ (s, 9H), 2.31 (s, 3H).

${ }^{13} \mathrm{C}$ NMR $\left(100 \mathrm{MHz}, \mathrm{MeOD}-\mathrm{d}_{4}\right): \delta=8.4\left(\mathrm{CH}_{3}\right), 26.8$ $\left(\mathrm{CH}_{3}\right), 27.6\left(2 \times \mathrm{CH}_{3}\right), 39.3(\mathrm{C}), 154.1(\mathrm{C}), 182.5(\mathrm{CO})$.

Anal for $\mathrm{C}_{7} \mathrm{H}_{12} \mathrm{~N}_{4} \mathrm{O}$ : C, 49.99; $\mathrm{H}, 7.19 ; \mathrm{N}, 33.31$. Found: C, 49.96; H, 7.14; N, 33.33.
1-(5-benzhydryl-1H-tetrazol-1-yl)-2,2-dimethylpropan-1-one (1f)

White solid; yield: $90 \%$ (1.58 g); mp: $154-158^{\circ} \mathrm{C}$.

${ }^{1} \mathrm{H}$ NMR (400 MHz, MeOD-d $\left.\mathrm{d}_{4}\right): \delta=1.07$ (s, 9H), 5.80 (s, 1H), 7.23-7.38 (m, 10H).

${ }^{13} \mathrm{C}$ NMR (100 MHz, MeOD-d $\left.\mathrm{d}_{4}\right): \delta=26.8,27.5,27.6$

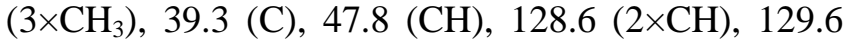
(4×CH), $129.9(4 \times \mathrm{CH}), 140.8(2 \times \mathrm{C}), 159.9(\mathrm{C}), 182.5$ $(\mathrm{C}=\mathrm{O})$.

Anal for $\mathrm{C}_{19} \mathrm{H}_{20} \mathrm{~N}_{4} \mathrm{O}$ : C, 71.23; $\mathrm{H}, 6.29 ; \mathrm{N}, 17.49$. found: C, 71.24; H, 6.26; N, 17.445 .

\section{Naphthalene-Catalysed Lithiation of Compounds 1. Preparation of Products 2. General Procedure}

A solution of the protected tetrazoles $1(0.5 \mathrm{mmol})$ in THF ( $2 \mathrm{~mL}$ ) was dropwise added to a green suspension of lithium powder (70 mg, $10 \mathrm{mmol}$ ) and naphthalene (26 mg, $0.2 \mathrm{mmol})$ in THF (5 mL), under Ar, at $0{ }^{\circ} \mathrm{C}$. After stirring at the same temperature for the time indicated in Table 1, methanol ( $5 \mathrm{~mL}$ ) was carefully added, the cooling bath was removed and the reaction was stirred till it reached room temperature. The free tetrazoles $\mathbf{2}$ were obtained in pure form after extraction with EtOAc, in the yields indicated in Table 1. The obtained tetrazoles 2 were characterised by comparison of their physical and spectroscopic data with the ones of the tetrazoles that we had previously synthesized.

\section{Acknowledgements}

This work was financially supported by

The A.N.D.R.S (Agence Nationale pour le Développement de la Recherche en Santé) (Algérie) and the Department of Organic Chemistry of the University of Alicante (Spain)

We are very grateful to the Spanish Ministerio de Asuntos Exteriores y de Cooperación for a cooperation grant (AP/039112/11). We are very grateful to Dr. Rosa Ortiz for her valuable help.

\section{References}

(1) (a) Kunz, H.; Waldmann, H. In Comprehensive Organic Synthesis, Vol. 6; Trost, B. M.; Fleming, I.; Winterfeldt, E., Eds.; Pergamon Press: Oxford, 1991, Chapter 3.1. (b) Greene, T. W.; Wuts, P. G. M. Protective Groups in Organic Synthesis, 3rd ed.; John Wiley \& Sons: New York, 1999. (c) Kocienski, P. J. Protecting Groups; Thieme: Stuttgart, 2004.

(2) See, for instance: Fernández, A.-M.; Plaquevent, J.-C.; Duhamel, L. J. Org. Chem. 1997, 62, 4007.

(3) See, for instance: Pinnick, H. W.; Fernández, E. J. Org. Chem. 1979, 44, 2810.

(4) Behloul, C.; Guijarro, D.; Yus, M. Synthesis. 2006, 309.

(5) See, for instance: (a) Nicolaou, K. C.; Webber, S. E. Synthesis 1986, 453. (b) Danishefsky, S. J.; Armistead, D. M.; Wincott, F. E.; Selnick, H. G.; Hungate, R. J. Am. Chem. Soc. 1989, 111, 2967.

(6) Horner, L.; Neumann, H. Chem. Ber. 1965, 98, 3462.

(7) See, for instance: Reference 1c, p. 416. 
(8) See, for instance: Srimurugan, S.; Suresh, P.; Babu, B.; Hiriyanna, S. G.; Pati, H. N. Chem. Pharm. Bull. 2008, 56, 383.

(9) For reviews, see: (a) Yus, M. Chem. Soc. Rev. 1996, 25, 155. (b) Ramón, D. J.; Yus, M. Eur. J. Org. Chem. 2000, 225. (c) Yus, M. Synlett. 2001, 1197. (d) Yus, M.; Ramón, D. J. Latv. J. Chem. 2002, 79. (e) Ramón, D. J.; Yus, M. Rev. Cubana Quim. 2002, 14, 76. (f) Yus, M. In The Chemistry of Organolithium Compounds; Rappoport, Z.; Mareck, I., Eds.; J. Wiley \& Sons: Chichester, 2004, Chapter 11.

(10) For mechanistic studies, see: (a) Yus, M.; Herrera, R. P.; Guijarro, A. Tetrahedron Lett. 2001, 42, 3455. (b) Yus, M.; Herrera, R. P.; Guijarro, A. Chem. Eur. J. 2002, 8, 2574. (c) Herrera, R. P.; Guijarro, A.; Yus, M. Tetrahedron Lett. 2003, 44, 1309.

(11) For a polymer version of this reaction, see: (a) Gómez, C.; Ruiz, S.; Yus, M. Tetrahedron Lett. 1998, 39, 1397. (b) Gómez, C.; Ruiz, S.; Yus, M. Tetrahedron 1999, 55, 7017. (c) Yus, M.; Gómez, C.; Candela, P. Tetrahedron 2002, 58, 6207. (d) Candela, P.; Gómez, C.; Yus, M. Russ. J. Org. Chem. 2004, 40, 795.

(12) This methodology has been extensively used in our laboratories for the following applications: generation of organolithium compounds from non-halogenated materials, ${ }^{12 a}$ preparation of very sensitive functionalised organolithium compounds, ${ }^{12 \mathrm{~b}-\mathrm{g}}$ reductive ring-opening of heterocycles, ${ }^{12 \mathrm{~h}-\mathrm{j}}$ generation of polylithium synthons ${ }^{12 k, 1}$ and for the activation of other metals, ${ }^{12 \mathrm{~m}}$ especially nickel. ${ }^{12 \mathrm{n}, \mathrm{o}}$ For reviews from our group, see: (a) Guijarro, D.; Yus, M. Recent Res. Dev. Org. Chem. 1998, 2, 713. (b) Nájera, C.; Yus, M. Trends Org. Chem. 1991, 2, 155. (c) Nájera, C.; Yus, M. Recent Res. Dev. Org. Chem. 1997, 1, 67. (d) Nájera, C.; Yus, M. Curr. Org. Chem. 2003, 7, 867. (e) Nájera, C.; Sansano, J. M.; Yus, M. Tetrahedron 2003, 59, 9255. (f) Chinchilla, R.; Nájera, C.; Yus, M. Chem. Rev. 2004, 104, 2667. (g) See also the especial issue of Tetrahedron Symposium in Print devoted to "Functionalised Organolithium Compounds” (Nájera, C.; Yus, M., Guest Eds.), Tetrahedron 2005, 61, 3125. (h) Yus, M.; Foubelo, F. Rev. Heteroa tom. Chem. 1997, 17, 73. (i) Yus, M.; Foubelo, F. Targets Heterocycl. Syst. 2002, 6, 136. (j) Yus, M. Pure Appl. Chem. 2003, 75, 1453. (k) Foubelo, F.; Yus, M. Trends Org. Chem. 1998, 7, 1. (l) Foubelo, F.; Yus, M. Curr. Org. Chem. 2005, 9, 459. (m) Guijarro, A.; Gómez, C.; Yus, M. Trends Org. Chem. 2000, 8, 65. (n) Alonso, F.; Radivoy, G.; Yus, M. Russ. Chem. Bull. 2003, 52, 2563. (o) Alonso, F.; Yus, M. Chem. Soc. Rev. 2004, 33, 284.

(13) Yus, M.; Behloul, C.; Guijarro, D. Synthesis 2003, 2179.

(14) Behloul, C.; Guijarro, D.; Yus, M. Synthesis 2004, 1274.

(15) Behloul, C.; Guijarro, D.; Yus, M. Tetrahedron 2005, 61, 6908.

(16) (a) Behloul, C.; Guijarro, D.; Yus, M. Tetrahedron 2005, 61, 9319. (b) Behloul, C.; Guijarro, D.; Yus, M. ARKIVOC 2007, vii, 41.

(17) Behloul, C.; Guijarro, D.; Yus, M. Synthesis 2006, 309.

(18) Yus, M.; Martínez, P.; Guijarro, D. Tetrahedron 2001, 57, 10119.

(19) Watson, S. C.; Eastham, J. F. J. Organomet. Chem. 1967, 9, 165.

(20) Koguro, K.; Oga, T.; Mitsui, S.; Orita, R. Synthesis. 1998, 910.

(21) Demko, Z. P.; Sharpless, K. B. J. Org. Chem. 2001, 66, 7945-7950. 
Reductive Removal of the Pivaloyl Protecting Group from Tetrazoles by a Naphthalene-Catalysed Lithiation Process

Cherif Behloul, ${ }^{* a}$ Aicha Chouti, ${ }^{a}$ David Guijarro, ${ }^{\mathrm{b}}$ Carmen Nájera ${ }^{\mathrm{b}}$ and Miguel Yus ${ }^{\mathrm{b}}$

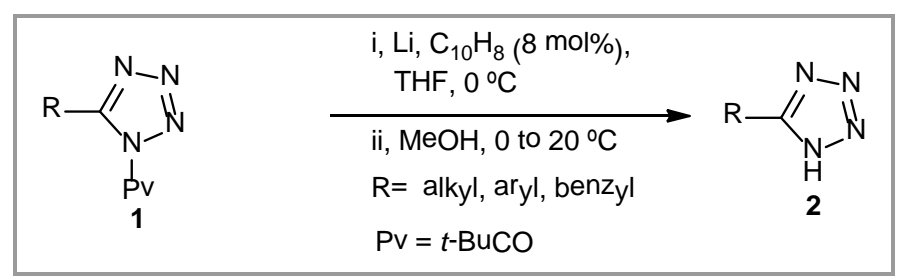

